\title{
BMJ Open Association of cultural origin and migration status with work-related mental health of migrants and refugees in Europe: a systematic review protocol
}

\author{
Regina Herold (D , ${ }^{1}$ Frederik Wuchenauer, ${ }^{1}$ Anja Kandler, ${ }^{1}$ Eva Morawa, ${ }^{1}$ \\ Susanne Unverzagt (D) ,2 Amanda Voss, ${ }^{3}$ Yesim Erim ${ }^{1}$
}

To cite: Herold R, Wuchenauer F, Kandler A, et al. Association of cultural origin and migration status with work-related mental health of migrants and refugees in Europe: a systematic review protocol. BMJ Open 2022;12:e052395. doi:10.1136/ bmjopen-2021-052395

- Prepublication history and additional supplemental material for this paper are available online. To view these files, please visit the journal online (http://dx.doi.org/10.1136/ bmjopen-2021-052395).

Received 14 April 2021 Accepted 06 December 2021

Check for updates

(C) Author(s) (or their employer(s)) 2022. Re-use permitted under CC BY-NC. No commercial re-use. See rights and permissions. Published by BMJ.

${ }^{1}$ Department of Psychosomatic Medicine and Psychotherapy, University Hospital of Erlangen, Friedrich-Alexander University Erlangen-Nürnberg (FAU),

Erlangen, Germany

${ }^{2}$ Center of Health Sciences, Institute of General Practice and Family Medicine, Martin-Luther University of Halle-Wittenberg, Halle, Germany

${ }^{3}$ Institute and Outpatient

Clinic of Occupational, Social, and Environmental Medicine,

Friedrich-Alexander University

Erlangen-Nürnberg (FAU),

Erlangen, Germany

Correspondence to

Ms Regina Herold;

regina.herold@uk-erlangen.de

\section{ABSTRACT}

\section{Introduction}

Migrants make up a significant proportion of the European working population. Previous studies have already shown that migrants and refugees often suffer from poor workrelated conditions in the host country, which might have an impact on mental health. Thus, the main objective of this systematic review is to analyse and summarise existing research on work-related conditions of migrants and refugees in Europe and to investigate the relationship of these conditions with their mental health.

Methods and analysis Three electronic databases (PubMed/MEDLINE, PsycINFO and CINAHL) will be systematically searched for eligible articles using quantitative study designs (randomised controlled trials, cohort, case-control and cross-sectional studies with and without control groups) written in English, German, French, Italian, Polish, Spanish or Turkish and published from 1st January 2016 onwards. The primary health outcomes will be diagnosed psychiatric and psychological disorders, suicide and suicide attempts, psychiatric and psychological symptoms, and perceived distress. The secondary health outcomes will be more general concepts of mental health such as well-being, life satisfaction and quality of life. Outcome measures must have been assessed by validated questionnaires. Screening of all articles, reference lists of included studies and relevant reviews as well as data extraction will be performed independently by two review authors. Methodological quality of primary studies will be assessed and discussed. The results of the primary studies will be summarised descriptively. Migrants and natives, migrants and refugees, migrants of different cultural backgrounds and migrants living in different host countries will be compared in terms of the association between their work-related conditions and their mental health.

Ethics and dissemination This systematic review is excluded from ethical approval because it will use previously approved published data from primary studies. The results of this review will be submitted to a related peer-reviewed journal.

PROSPERO registration number CRD42021244840.

\section{INTRODUCTION}

In recent years, there has been a substantial increase in global migration. In 2019,
Strengths and limitations of this study

- This systematic review about the association of work-related conditions and mental health of migrants and refugees in Europe will be based on a detailed search strategy including studies from European countries published in the last 5 years in seven European languages.

- Screening of articles as well as data extraction and methodological quality assessment using an appropriate validated tool will be performed independently by two review authors to minimise the probability of personal biases.

Due to time and language limitations, not all relevant studies might be found.

- The heterogeneity of self-report measures of mental health and work-related conditions complicates the direct comparability of primary studies.

272 million people were considered migrants. Two years earlier, among those having migrated internationally, 29 million represented as refugees and asylum seekers. ${ }^{1}$ According to The United Nations Recommendations on Statistics of International Migration, an 'international migrant' is defined as a person who has changed his or her country of residence. ${ }^{2}$ These individuals emigrate internationally for reasons such as work, family or study, whereas individuals who had to leave their homes due to conflict, persecution or catastrophes are referred to as refugees. Persons who have not yet been granted official refugee status are defined as asylum seekers. ${ }^{3}$ With the main migration destinations being high-income countries, ${ }^{4}$ Europe represents one of the regions with the highest number of migrants. Almost every third migrant worldwide lives in Europe. Approximately $11 \%$ of the population in European countries can be considered migrants. ${ }^{1}$ Some countries in Europe 
even have a significantly higher percentage of migrants in their society. Switzerland leads with $29.9 \%$ of migrants, followed by Sweden with 20\%, Austria with $19.9 \%$ and Belgium with $17.2 \% .^{5}$ A closer look at the countries of origin of migrants and refugees/asylum seekers reveals a very heterogeneous picture. While migration from one European country to another can be described as the largest migration corridor worldwide, 2019 also saw large inflows of migrants from Northern and Western Africa, Central and Southern Asia, Latin America and the Caribbean and sub-Saharan Africa. ${ }^{1}$ All those migrants make up a not insignificant part of the working population in Europe. ${ }^{4}$ Several studies have already shown that migrants and refugees/asylum seekers are considered a particularly vulnerable group in terms of mental health in European host countries. Migrants reported higher levels of post-traumatic stress disorder (PTSD) ${ }^{6}$ as well as lower general and mental health ${ }^{7}$ compared with natives. Among refugees and asylum seekers, a similar phenomenon emerged. Studies indicated higher prevalence rates of mental disorder symptoms (PTSD and depression) among refugees and asylum seekers than natives. ${ }^{689}$ Due to their high mental distress, vocational factors prevalent in the host country that could have an impact on the mental health of migrants and refugees/asylum seekers in Europe should be detected.

Various studies have already examined the working conditions that migrants are confronted with in their host country worldwide. These studies have often revealed disadvantages in labour market integration such as difficulties in finding a job which ultimately leads to higher unemployment rates for migrants. ${ }^{10}$ Moreover, it became clear that migrants often suffer from worse working conditions than their autochthonous counterparts. For example, they are often hired in jobs that are considered low-skilled ${ }^{7}$ and compensated with a low income. ${ }^{11}$ Furthermore, they are more likely to be exposed to various health hazards in the workplace, such as pesticides and chemicals and show increased potential for injury. ${ }^{11-14}$ Migrants from low-income and middle-income countries seem to be particularly vulnerable. ${ }^{15}$ Further, migrant workers are more likely to experience exploitation and abuse, ${ }^{11}$ as well as physical, psychological and sexual violence, ${ }^{12}$ with women being at particular risk. ${ }^{11}$ These negative working conditions affect both the physical and mental health of migrants. ${ }^{12}{ }^{15}$ In terms of mental well-being, mental health disadvantages such as psychotic, neurotic and mood disorders were identified. ${ }^{12}$ Other studies, however, found no meaningful difference in working conditions between migrant and autochthonous workers. ${ }^{16}$ Due to the inconsistent findings, it is of upmost importance to look more deeply into work-related conditions of migrants and refugees/asylum seekers.

The already mentioned very heterogeneous regions of origin of migrants and refugees/asylum seekers in Europe represent countries of very different cultural backgrounds, all of which converge in the destination region. These cultural differences might lead to difficulties in living together in a common destination country. One of the most relevant classifications of cultural backgrounds is offered by Hofstede's categorisation of collectivist societies and individualist societies. Individualistic cultures are characterised by relatively loose ties between individuals, whereas in collectivistic cultures life tends to take place in strong, cohesive in-groups. ${ }^{17}$ Individualism is more prevalent in Western and developing countries, while collectivism is more common in less developed and Eastern countries. ${ }^{18}$ However, cultural origin not only has an influence on life in society, but also on working life. For example, an experimental study by Earley ${ }^{19}$ showed that collectivist subjects performed best when they worked in a group and anonymously, while they performed weakest when they worked alone and their performance was traceable. Subjects with individualistic backgrounds, however, performed best when they worked alone and their performance was traceable. Their performance was very weak when working in a group and when their performance was not comprehensible. ${ }^{19}$ Furthermore, differences in the quality and level of education in non-European countries in contrast to European countries ${ }^{20}{ }^{21}$ might influence the chances to receive a desirable employment in the host country. Accordingly, professional training from a non-European country might lead to worse work-related conditions. Additionally, research has already shown that diverse cultural backgrounds can function as a risk factor for developing mental health burdens. For example, studies comparing respondents with more individualistic cultural backgrounds with respondents originating from more collectivistic societies showed that individuals from individualistic countries experienced more mental complaints. ${ }^{22}{ }^{23}$ Furthermore, there is general consensus among researchers that persons from different cultures show different preferences of certain psychological symptoms and disorders. Individuals from traditional collectivist societies, who favour social harmony as well as traditions and emotional dependence on society, are more likely to exhibit somatic symptoms instead of disorders such as depression and PTSD. ${ }^{22} 24$ This justifies the question whether cultural differences can also lead to differences in emotional satisfaction at work. These cultural differences due to origin highlight the different needs and statuses of people of different cultural origins that have to be addressed in preventing the development of mental health problems among migrant workers in Europe. In conclusion, we will shed more light on differences in work-related conditions and their relationship with mental health among migrants and refugees/asylum seekers of different cultural backgrounds.

In terms of distinguishing people of different cultural backgrounds, the question also arises about the extent to which migrant and refugee/asylum seeker workers differ in terms of work-related conditions and their relationship to mental health as the population of refugees/asylum seekers represents a special group of migrants. Refugees and asylum seekers can be seen as a specific migrant group because, by definition, they have different premigratory 
and postmigratory conditions ${ }^{25}$ than general migrants due to the sometimes traumatic refugee experience ${ }^{26}$ itself and the subsequent asylum process, ${ }^{27}$ making them differently vulnerable to mental health problems. ${ }^{25-27}$ In line with this, various previous studies have shown that refugees suffer significantly more often from mental illnesses such as depression, anxiety and PTSD than voluntarily migrated labour migrants. ${ }^{28}$ Due to the greater psychological strain on refugees and asylum seekers, it is reasonable to assume that this particular subgroup could suffer particularly from poor working conditions. However, the mental burden of refugees/asylum seekers due to their working conditions has rarely been studied. Nevertheless, the findings show that differences exist between migrants and refugees in labour market outcomes. Refugees are characterised by their disadvantages in the work context in comparison to autochthones and other migrant groups. ${ }^{29}$ For example, Jamil et al showed that refugees were twice more often affected by unemployment than migrants in general. ${ }^{30}$ This shows that migrants and refugees/asylum seekers hold different positions in the labour market. Accordingly, migrants and refugees/asylum seekers must be considered separately with regard to their working conditions and their influences on mental health. There should be a targeted search for differences between subgroups in order to specifically uncover potential grievances in terms of working conditions and take action against them. The only way to ensure successful assistance to maintain or improve the mental health of migrants and refugees/asylum seekers is to adapt it to the needs of these subgroups.

Whereas the member states of the European Union (EU) have generally taken a similar direction with regard to migration and integration policy, ${ }^{31}$ they clearly differ from other Western countries such as the so-called classic immigration countries USA, Canada and Australia. ${ }^{32}$ Although some research groups have already engaged in the study of work-related conditions and their influence on the mental well-being of migrants worldwide, to our knowledge, no systematic review exists in this area with a specific European focus. Thus, there is a need to better understand and systematically evaluate the association of work-related conditions and the mental health of migrants and refugees/asylum seekers in Europe. For this reason, this systematic review will focus only on European countries. Despite the fact that there has been a common tightening of integration and immigration policies within all member states of the EU, European countries partially differ in terms of migration policies, depending on the influence of heterogeneous migration histories. France, Germany, Austria and Belgium, for example, count as 'traditional migration countries', whereas Italy, Greece and the Czech Republic have experienced greater immigration flows only since the 1990s. These differences could have an impact on the working lives of migrants and refugees/asylum seekers in the country of residence. ${ }^{31}$ Due to the potential differences in the immigration policies of the individual EU-member states and since it can be assumed that non-member states in Europe may have developed even more independently with regard to their migration policies, it is of interest to also compare working conditions of migrants and refugees/asylum seekers and their influence on mental health between the individual European host countries.

Increased migration to Europe, as well as the facts that migrants and refugees are considered a population group being at particular risk of mental burden and make up a non-negligible portion of the workforce in Europe, highlight the importance of more in-depth research on work-related conditions and their influence on the mental health of migrants and refugees/asylum seekers in Europe with special consideration to their cultural backgrounds and migration status (voluntarily migrated migrants vs involuntarily migrated refugees and asylum seekers). This is necessary to detect and actively address potential abuses in the working context in order to maintain and, if necessary, restore the mental health of migrants and refugees/asylum seekers.

\section{OBJECTIVES}

The main objective of our planned systematic review is to analyse and summarise the (1) association between workrelated conditions and mental health of migrants and refugees and asylum seekers (both summarised as 'refugees' in the following) in Europe.

To broaden understanding of this association we will further (2) describe the kinds of work-related conditions of migrants and refugees in Europe and (3) compare them to autochthonous workers. Furthermore, we will examine (4) if migration experience (migrants/refugees vs natives) influences the association of work-related conditions and mental health and (5) if migration status (migrants vs refugees) influences the association between work-related conditions and mental health. Additionally, we will (6) compare migrants and refugees of different cultural backgrounds in Europe in terms of the association between work-related conditions and mental health and (7) compare migrants and refugees living in different host countries.

\section{METHODS AND ANALYSIS}

The content and structure of this study protocol is developed in accordance with the reporting guidance 'Preferred Reporting Items for Systematic Reviews and Meta-Analyses (PRISMA) Protocols statement'. ${ }^{33}$ Significant protocol amendments will be announced with the publication of the systematic review.

\section{Eligibility criteria}

The eligibility criteria of the included studies will be described based on the Population, Exposure, Comparator and Outcome framework.

Types of population

We will include studies of internationally migrated firstgeneration migrants, refugees and asylum seekers of 
working age (15-70 years). Participants must be currently working and residing in an European country. Migrants in irregular migration situations and migrants who are informally employed will also be encluded. We will explicitly exclude studies of internal migrants or refugees, secondgeneration migrants or refugees, migrants and refugees younger than 15 or older than 70 years, not currently working (which includes students and apprentices) and not living in European countries. If clear information regarding inclusion criteria is missing, the corresponding authors will be contacted. Studies that do not separate working age from non-working age participants will be excluded.

\section{Types of exposure}

We will include original studies of the association of any work-related conditions with the mental health of migrants and refugees in Europe. Work-related conditions include 'organizational conditions' (income level, formality of work, work contract, weekly working hours, shift work, overqualification, ...), 'social conditions at work' (discrimination, prejudice, violence, leadership perceptions, sense of community, ...) or special issues such as 'postmigration stressors migrants and refugees in Europe are confronted with at the workplace ${ }^{, 34}$ (language barriers, cultural differences, ...).

\section{Types of comparators}

A comparison/control group is not obligatory. We will include studies comparing (1) migrant/refugee workers with autochthonous workers, (2) migrant workers with refugee workers, (3) migrant and refugee worker groups of different cultural origins, (4) migrant or refugee workers living and working in diverse European host countries and (5) burdened with unburdened workers regarding their work-related conditions and mental health outcomes. Furthermore, studies might also compare workers with different characteristics of workrelated conditions in relation to their mental health.

\section{Types of outcomes}

The primary outcomes of interest are psychiatric and psychological symptoms, perceived distress, diagnosed psychiatric and psychological disorders or suicide or attempted suicide. The secondary mental health outcomes are indicators of well-being such as quality of life and life satisfaction. The primary outcomes will be categorised as follows: cluster A: psychological and psychiatric diagnoses (including suicide and suicide attempts), cluster B: psychological and psychiatric symptoms (such as anxiety, depression, PTSD, somatoform disorders) and cluster C: general distress. The secondary outcomes will be classified as cluster D: more general related constructs of mental health (well-being, quality of life, life satisfaction). If needed, there will be the additional cluster $\mathrm{E}$, which includes all outcomes that cannot be assigned to clusters A-D. Only studies examining at least one migrants' or refugees' mental health outcome with a validated measure will be included. Translated measurements in order to quantify migrant or refugee workers' mental health must have been at least validated in the original language.

\section{Study design}

We will include quantitative studies such as randomised controlled trials, cohort studies, case-control studies and cross-sectional studies with and without control groups. We will explicitly exclude qualitative studies and case studies. Reviews will be screened for relevant references before exclusion. Studies focusing on migrants' and refugees' work-related conditions only as well as studies focusing on migrants' and refugees' mental health only, without any consideration to the association between work-related conditions and mental health outcomes, will be excluded.

\section{Setting and time frame}

No restrictions by the type of setting will be used. Studies published between 1 January 2016 and 16 March 2021 will be included.

\section{Language}

Studies written in English, German, French, Italian, Polish, Spanish and Turkish will be included.

\section{Publication status}

Studies published in peer-reviewed journals will be included. Unpublished studies as well as editorials, letters, 'grey literature' such as conference abstracts, dissertations and non-peer review articles will be excluded. Only full-text versions of relevant studies (assessed by using the 'Strengthening the Reporting of Observational Studies in Epidemiology Statement ${ }^{35}$ ) will be considered.

\section{Information sources}

The three most relevant electronic databases for this research area (PubMed/Medline, PsycINFO and CINAHL) will be systematically searched for relevant literature. Additionally, the reference lists of included studies and relevant reviews will be searched.

\section{Search strategy}

The search terms and syntax include combinations of MeSH Terms, where applicable, and title/abstract text terms based on the eligibility criteria. According to the eligibility criteria the strategy includes three search term clusters: (1) terms related to the study population such as "migrant*" or "refugee*", (2) terms related to workrelated conditions such as "employ*" or "work*" and (3) terms related to mental health outcomes such as "mental disorder*" or "well-being".

In the following, an exemplary search syntax is shown based on the search in PubMed: (" "transients and migrants" [MeSH Terms] OR "emigrants and immigrants" [MeSH Terms] OR "refugees" [MeSH Terms] OR "ethnic groups" [MeSH Terms] OR "minority groups"[MeSH Terms] OR "human 
migration" [MeSH Terms] OR “migra*“[Title/Abstract] OR "emigra*"[Title/Abstract] OR "immigra*"[Title/ Abstract] OR "refugee*"[Title/Abstract] OR "asylum seeker*"[Title/Abstract] OR "foreign*"[Title/Abstract] OR "resettl*"[Title/Abstract] OR "guest worker*"[Title/ Abstract] OR "displaced person*"[Title/Abstract]) AND ("employment"[MeSH Terms] OR "work"[MeSH Terms] OR "occupations"[MeSH Terms] OR "employ*"[Title/ Abstract] OR "work*“[Title/Abstract] OR "occupation*"[Title/Abstract] OR "vocation*"[Title/Abstract] OR “jobs"[Title/Abstract] OR "job"[Title/Abstract]) AND ("mental health" [MeSH Terms] OR "mental disorders" [MeSH Terms] OR "stress, psychological" [MeSH Terms] OR "mental fatigue" [MeSH Terms] OR "depressive disorder" [MeSH Terms] OR "depression" [MeSH Terms]) OR "quality of life" [MeSH Terms] OR "wellbeing"[Title/Abstract] OR "wellbeing"[Title/Abstract] OR "quality of life"[Title/Abstract] OR "life satisfaction"[Title/Abstract] OR "mental*“[Title/Abstract] OR "psychiatric*"[Title/Abstract] OR "psychological*“[Title/Abstract] OR "anxi*"[Title/Abstract] OR "depress*“[Title/Abstract] OR "burnout"[Title/ Abstract] OR "burn-out"[Title/Abstract] OR "somatization"[Title/Abstract] OR "somatisation"[Title/ Abstract] OR "somatoform"[Title/Abstract] OR "pain disorder"[Title/Abstract] OR "somatic symptom disorder"[Title/Abstract] OR "suicid*"[Title/Abstract] OR "insomnia"[Title/Abstract] OR "emotional stress" [Title/Abstract] OR “distress"[Title/Abstract])) NOT ("USA"[Title/Abstract] OR "United States"[Title/ Abstract] OR "Canada" [Title/Abstract] OR "Australia"[Title/Abstract] OR "migraine"[Title/Abstract] OR "cancer"[Title/Abstract] OR "qualitative stud*"[Title/ Abstract]) Filters: English, French, German, Italian, Polish, Spanish, Turkish, from 2016/1/1 - 2021/3/31. The exact search strategies for the other two electronic databases can be found in online supplemental file 1 .

\section{Study records}

Data management

EndNote V.X ${ }^{36}$ and an Excel spreadsheet will be used for data management.

\section{Data collection, selection process and extraction}

After identification and exclusion of duplicates two review authors will independently screen the titles and abstracts of the publications based on the inclusion and exclusion criteria. The full texts of the relevant articles will then independently be assessed by the two reviewers to decide whether they meet the inclusion criteria. The reference lists of review articles and the included publications will be scanned for relevant articles missed in the initial search. Any disagreements will be discussed between the two reviewers and if an agreement cannot be reached a third/fourth reviewer will be consulted. A PRISMA flow chart ${ }^{33}$ showing details of studies included and excluded at each stage of the study selection process will be provided.

\section{Data items}

From each included study the two review authors will independently extract data on the following variables:

- Study characteristics: study authors, publication year, study design, country.

- Population characteristics: sample size with gender distribution, information about the existence of a control group and the type of group(s) (migrant workers vs native workers, migrant workers vs refugee workers, workers of different cultural origins, workers living and working in different European host countries) and size of group(s), migrants' and refugees' countries of origin.

- Exposure: kinds of work-related conditions and measurement instrument, if available.

- Outcome: primary and secondary mental health indices and measurement instrument.

- Main results of the study.

In case of disagreement between the two authors, a third/fourth reviewer will be consulted.

\section{Risk of bias in individual studies}

The two review authors will independently evaluate the quality of research papers using the 'Newcastle Ottawa Quality Assessment Scale, ${ }^{37}$ for cohort studies, casecontrol studies and cross-sectional studies. Finally, each study will receive a score for low (0-3 points), moderate (4-6) or high quality (7-9). In the case of relevant randomised controlled trials the revised 'Cochrane riskof-bias tool' $(\operatorname{RoB} 2)^{38}$ will be used. Disagreements will be first discussed between the two reviewers. If agreement will not be reached, a third/fourth reviewer will be consulted.

Additionally, the validity of the used mental health measure will be assessed by stating whether a validated questionnaire was used in the original language or whether only the translation of such a measure or a validated or a cultural adapted version was used.

\section{Data analysis and synthesis}

One review author will carry out a narrative (descriptive) synthesis of the included studies following the PRISMA checklist $^{33}$ (see above). No formal quantitative analysis will be conducted. As part of the synthesis the quality assessment will be discussed, thereby studies of poor quality will be identified. Their potential impact on the overall results will be discussed. Based on the synthesis, the extent to which work-related conditions differ between workers of different origins and the extent to which they affect mental health will be shown. In addition, recommendations will be presented on how to improve workrelated conditions for the vulnerable group of migrants and refugees in Europe to guarantee their psychological well-being and their working capability.

If relevant studies focusing on subgroups will be found, comparisons will descriptively be conducted. If possible, we will compare migrants' and refugees' work-related conditions with natives' work-related conditions as well 
as their impact on the mental health of both subgroups. Furthermore, we will present differences or similarities of work-related conditions and their impact on mental health between migrants and refugees, if possible. Additionally, we will report differences or similarities of work-related conditions and their impact on mental health between migrant/refugee workers of different cultural origins by categorising their countries of origins according to the theory of collectivism and individualism by Hofstede. ${ }^{17}$ Finally, due to differences in migration and integration laws between European countries, ${ }^{31}$ the working conditions and their impact on the mental health of migrants and refugees living and working in different European host countries will be compared.

\section{Ethics and dissemination}

This systematic review will be submitted to a leading journal in this field.

\section{DISCUSSION}

This systematic review will provide in-depth insight into the relationship between work-related conditions and their influence on the mental health of migrants and refugees in Europe. Taking into account current quantitative research, this systematic review will address the following research questions: It will provide information (1) on the types of work-related conditions migrants and refugees face in the labour market in Europe, and the extent to which these conditions impact mental health. Furthermore, it will be shown (2) whether migrants and refugees work under different work-related conditions than autochthonous workers and (3) to what extent migrants and refugees differ compared with natives regarding the relationship between work-related conditions and mental health. In addition, the study will examine (4) whether migrants and refugees (5) as well as migrants and refugees of different cultural backgrounds differ in terms of the relationship between work-related conditions and mental health. Lastly, (6) migrants and refugees living in different European host countries will be compared in terms of the association between their working conditions and mental health.

Some sources of bias risk can be identified in advance. Since 'grey literature' will be explicitly excluded, publication bias cannot be prevented. ${ }^{39}$ However, 'grey literature', especially conference papers, often do not provide precise details to be useful for screening relevant research, ${ }^{40}$ thus not too much methodological loss is to be expected here.

Furthermore, the exclusion of primary studies published before 2016 could introduce the problem of not finding important studies published in the years before. However, since there was a huge growth of asylum seekers migrating to Europe since 2014 (but more so in 2015 and 2016), ${ }^{41}$ we decided to limit the inclusion of relevant studies to this period. That is, because from 2016 onwards the direct impact of refugee movements on the labour market can be captured and thus the scientific data reflect the current situation.

Since we cannot include all primary studies regardless of language, the problem might arise that not all relevant studies may be found. However, since we have the ability to include studies in seven different languages, we cover a wide range of relevant primary studies, especially since our language skills coincide with common languages in the European region and it can be assumed that relevant studies might be published mainly in these languages.

When looking at the methods of measurement for mental health, it is apparent that a large variety of constructs as well as methods will be incorporated. These include self-reported measures of both psychopsychiatric symptoms or medical diagnoses and more general constructs such as well-being or quality of life or, in some circumstances, suicide rates or attempted suicide. However, by explicitly excluding non-validated measurement instruments to assess mental health, health outcomes can be compared quite well. Nevertheless, it must be noted that self-reported measures should always be viewed critically due to bias susceptibility because of social desirability or specific response tendencies of the participant. ${ }^{42}$ With regard to workrelated conditions, however, it must be noted that no validated measures must have been used in the primary studies. This complicates the comparability of the data. However, since our aim is also to report descriptively on work-related conditions, the high variety of different work-related conditions due to the very different assessments should be seen as an advantage rather than a disadvantage.

The connection between stress at work and mental health has been perceived very clearly in recent years. ${ }^{7}$ Workplace-related stress and mental disorders resulting hereof lead to many days of absence from work in Western countries. ${ }^{43}$ Measures should be developed to improve conditions at the workplace and thus minimise the causes for mental disorders, or to offer low-threshold psychotherapies directly at the workplace. Therefore, it is of upmost importance to be informed about the specific conditions and distress of immigrants in the workplace as migrants and refugees make up a not insignificant part of the working population in European society. ${ }^{4}$ This systematic review will increase our insights in this context and thereby also contribute to the optimisation of conditions at the workplace and mental health.

To our knowledge, this planned systematic review will be the first to examine work-related conditions and mental health of migrants and refugees, comparing both population groups while additionally considering cultural backgrounds and paying special attention to the healthcare sector.

Acknowledgements We would like to express our sincere thanks to Dr. Eva Rothermund, Department of Psychosomatic Medicine and Psychotherapy, University of Ulm, for her valuable expertise. 
Contributors YE conceived the initial idea for the review. RH, FW, AK, EM and YE designed the review. SU participated in study design. AV provided expert input on theoretical considerations. $\mathrm{RH}$ drafted the manuscript. All the authors contributed to the revision of the manuscript and approved the final version. $\mathrm{RH}$ is the guarantor of the systematic review.

Funding The authors have not declared a specific grant for this research from any funding agency in the public, commercial or not-for-profit sectors.

Competing interests None declared.

Patient consent for publication Not applicable.

Provenance and peer review Not commissioned; externally peer reviewed.

Supplemental material This content has been supplied by the author(s). It has not been vetted by BMJ Publishing Group Limited (BMJ) and may not have been peer-reviewed. Any opinions or recommendations discussed are solely those of the author(s) and are not endorsed by BMJ. BMJ disclaims all liability and responsibility arising from any reliance placed on the content. Where the content includes any translated material, BMJ does not warrant the accuracy and reliability of the translations (including but not limited to local regulations, clinical guidelines, terminology, drug names and drug dosages), and is not responsible for any error and/or omissions arising from translation and adaptation or otherwise.

Open access This is an open access article distributed in accordance with the Creative Commons Attribution Non Commercial (CC BY-NC 4.0) license, which permits others to distribute, remix, adapt, build upon this work non-commercially, and license their derivative works on different terms, provided the original work is properly cited, appropriate credit is given, any changes made indicated, and the use is non-commercial. See: http://creativecommons.org/licenses/by-nc/4.0/.

\section{ORCID iDs}

Regina Herold http://orcid.org/0000-0002-9551-6661

Susanne Unverzagt http://orcid.org/0000-0002-0108-0415

\section{REFERENCES}

1 United Nations, Department of Economic and Social Affairs, Population Division, et al. International migration 2019: report. New York: United Nations, 2019.

2 United Nations DoEasA. Recommendations on statistics of international migration. Revision 1998;1.

3 McAuliffe M, Bauloz C, Nguyen M. Migration and migrants: A global overview. In: McAuliffe M, Khadria B, eds. World migration report 2020. Geneva, Switzerland: International Organization for Migration, 2019.

4 McAuliffe M, Khadria B. Report Overview: Providing perspective on migration and mobility in increasingly uncertain times. In: Gibson $\mathrm{M}$, ed. World migration report. Geneva, Switzerland: International Organization for Migration, 2019.

5 McAuliffe M, Kitimbo A, Abel G. Migration and migrants: Regional dimensions and developments. In: Gibson M, ed. World migration report 2020. Geneva, Switzerland: International Organization for Migration, 2019: 53-124.

6 Close C, Kouvonen A, Bosqui T, et al. The mental health and wellbeing of first generation migrants: a systematic-narrative review of reviews. Global Health 2016;12:47.

7 Arici C, Ronda-Pérez E, Tamhid T, et al. Occupational health and safety of immigrant workers in Italy and Spain: a scoping review. Int J Environ Res Public Health 2019;16:4416.

8 Blackmore R, Boyle JA, Fazel M, et al. The prevalence of mental illness in refugees and asylum seekers: a systematic review and meta-analysis. PLoS Med 2020;17:e1003337.

9 Giacco D, Priebe S. Mental health care for adult refugees in highincome countries. Epidemiol Psychiatr Sci 2018;27:109-16.

10 Uhlendorff A, Zimmermann KF. Unemployment dynamics among migrants and natives. Economica 2014;81:348-67.

11 Moyce SC, Schenker M. Migrant workers and their occupational health and safety. Annu Rev Public Health 2018;39:351-65.

12 Malhotra R, Arambepola C, Tarun S, et al. Health issues of female foreign domestic workers: a systematic review of the scientific and gray literature. Int J Occup Environ Health 2013;19:261-77.

13 Orrenius PM, Zavodny M. Do immigrants work in riskier jobs? Demography 2009;46:535-51.

14 Yanar B, Kosny A, Smith PM. Occupational health and safety vulnerability of recent immigrants and refugees. Int J Environ Res Public Health 2018;15:2004.
15 Hargreaves S, Rustage K, Nellums LB, et al. Occupational health outcomes among international migrant workers: a systematic review and meta-analysis. Lancet Glob Health 2019;7:e872-82.

16 Sterud T, Tynes T, Mehlum IS, et al. A systematic review of working conditions and occupational health among immigrants in Europe and Canada. BMC Public Health 2018;18:770.

17 Hofstede G. Dimensionalizing cultures: the Hofstede model in context. Online Readings in Psychology and Culture 2011;2:1-26.

18 Hofstede G, Hofstede GJ, Minkov M. Cultures and organizations - Software of the mind. 3rd ed. United States: The McGraw-Hill Professional, 2010: 1-576.

19 Earley PC. Social loafing and collectivism: A comparison of the United States and the People's Republic of China. Adm Sci Q 1989;34:565-81.

20 Helgesson M, Wang M, Niederkrotenthaler T, et al. Labour market marginalisation among refugees from different countries of birth: a prospective cohort study on refugees to Sweden. J Epidemiol Community Health 2019;73:407-15.

21 Ronda-Pérez E, Martínez JM, Reid A, et al. Longer residence of ecuadorian and colombian migrant workers in Spain associated with new episodes of common mental disorders. Int $J$ Environ Res Public Health 2019;16:2027.

22 Schubert CC, Punamäki R-L. Mental health among torture survivors: cultural background, refugee status and gender. Nord J Psychiatry 2011;65:175-82.

23 Wittig U, Lindert J, Merbach M, et al. Mental health of patients from different cultures in Germany. Eur Psychiatry 2008;23:s28-35.

24 Moisander PA, Edston E. Torture and its sequel-a comparison between victims from six countries. Forensic Sci Int 2003;137:133-40.

25 Porter M, Haslam N. Predisplacement and postdisplacement factors associated with mental health of refugees and internally displaced persons: a meta-analysis. JAMA 2005;294:602-12.

26 Heeren M, Wittmann L, Ehlert U, et al. Psychopathology and resident status - comparing asylum seekers, refugees, illegal migrants, labor migrants, and residents. Compr Psychiatry 2014;55:818-25.

27 Laban CJ, Gernaat HBPE, Komproe IH, et al. Impact of a long asylum procedure on the prevalence of psychiatric disorders in Iraqi asylum seekers in the Netherlands. J Nerv Ment Dis 2004;192:843-51.

28 Lindert J, Brahler E, Wittig U. Depressivität, Angst und posttraumatische Belastungsstörung bei Arbeitsmigranten, Asylbewerbern und Flüchtlingen. Systematische Übersichtsarbeit zu Originalstudien. Psychother Psychosom Med Psychol 2008;58:109-22.

29 Martín I, Arcarons A, Aumüller J. From refugees to workers: mapping labour market integration support measures for asylum-seekers and refugees in EU member states. Gütersloh, Germany: Bertelsmann Stiftung;2016:1-176

30 Jamil H, Kanno SS, Abo-Shasha R, et al. Promoters and barriers to work: a comparative study of refugees versus immigrants in the United States. New Iraqi J Med 2012;8:19-28.

31 Göbel B. In Vielfalt verbunden - Zusammenfassende Beobachtungen zur Integrationspolitik in Europa. Integrationskontinent Europa Integration von Zuwanderern im europäischen Vergleich. Berlin, Germany: Konrad-Adenauer-Stiftung e.V, 2019.

32 Hoesch K. Migration und Integration in internationaler Perspektive. Migration und Integration - Eine Einführung. Münster, Deutschland: Springer VS, 2018: 126-215.

33 Moher D, Liberati A, Tetzlaff J, et al. Preferred reporting items for systematic reviews and meta-analyses: the PRISMA statement. $J$ Clin Epidemiol 2009;62:1006-12.

34 Carlsson J, Sonne C. Mental health, pre-migratory trauma and post-migratory stressors among adult refugees. In: Nexhmedin M, Nickerson A, eds. Mental health of refugee and conflict-affected populations - Theory, research and clinical practice. Switzerland: Springer Nature, 2018: 15-35.

35 von Elm E, Altman DG, Egger M, et al. The strengthening the reporting of observational studies in epidemiology (STROBE) statement: guidelines for reporting observational studies. Int J Surg 2014;12:1495-9.

36 EndNote [program]. EndNote $X 9$ version. Philadelphia, PA: Clarivate, 2013.

37 Wells G, Shea B, O'Connell D. The Newcastle-Ottawa Scale (NOS) for assessing the quality of nonrandomised studies in meta-analyses, 2000. Available: http://www.ohri.ca/programs/clinical_epidemiology/ oxford.asp [Accessed 23 Dec 2021].

38 Sterne JAC, Savović J, Page MJ, et al. RoB 2: a revised tool for assessing risk of bias in randomised trials. BMJ 2019;366:14898. 
39 Scherer RW, Langenberg P, von Elm E. Full publication of results initially presented in abstracts. Cochrane Database Syst Rev 2007;2:MR000005.

40 O'Connor AM, Anderson KM, Goodell CK, et al. Conducting systematic reviews of intervention questions I: writing the review protocol, formulating the question and searching the literature. Zoonoses Public Health 2014;61:28-38.

41 Grote J. Die veränderte Fluchtmigration in den Jahren 2014 bis 2016: Reaktionen und Maßnahmen in Deutschland; Fokusstudie der deutschen nationalen Kontaktstelle für das Europäische Migrationsnetzwerk (EMN). Nürnberg, Germany: Bundesamt für Mlgration und Flüchtlinge (BAMF), 2018.

42 Furnham A, Henderson M. The good, the bad and the mad: response bias in self-report measures. Pers Individ Dif 1982;3:311-20.

43 Hansen H-T, Holmas TH, Islam MK, et al. Sickness absence among immigrants in Norway: does occupational disparity matter? Eur Sociol Rev 2014;30:1-12. 